\title{
Anabases
}

ANABASES Traditions et réceptions de l'Antiquité

5 | 2007

Varia

\section{Justinien visité et revisité}

\section{Pierre-Louis Malosse}

\section{OpenEdition}

Journals

Édition électronique

URL : http://journals.openedition.org/anabases/3215

DOI : 10.4000/anabases.3215

ISSN : 2256-9421

\section{Éditeur}

E.R.A.S.M.E.

\section{Édition imprimée}

Date de publication : 1 mars 2007

Pagination : 229-235

ISSN : 1774-4296

\section{Référence électronique}

Pierre-Louis Malosse, « Justinien visité et revisité », Anabases [En ligne], 5 | 2007, mis en ligne le 01 janvier 2012, consulté le 21 octobre 2019. URL : http://journals.openedition.org/anabases/3215 ; DOI : 10.4000/anabases.3215

Ce document a été généré automatiquement le 21 octobre 2019

(c) Anabases 


\title{
Justinien visité et revisité
}

\author{
Pierre-Louis Malosse
}

1 L'Antiquité tardive et Byzance ne sont pas les destinations favorites des voyageurs temporels. Ce n'est pas non plus la référence des œuvres relevant de ce sous-genre que la science-fiction a développé à partir du modèle fourni par Tolkien et qui se rapproche du merveilleux traditionnel, ce qu'on appelle en anglais fantasy. On préfère l'Antiquité classique ou le Moyen Âge occidental. Il est vrai que la culture des auteurs de sciencefiction est "classique », au sens propre, et que l'on n'étudie guère ces périodes dans les classes. Deux romans récemment parus en France font exception - à moins qu'ils ne soient les premiers témoins d'une vogue à venir, qui s'expliquerait peut-être par le regain d'intérêt que ces périodes longtemps délaissées suscitent depuis quelques années dans les lycées et les universités.

2 Mais je voudrais évoquer auparavant une œuvre plus ancienne, de Lyon Sprague de Camp, traduite en français sous le titre De peur que les ténèbres ${ }^{1}$ : Martin Padway, un doctorant américain d'histoire ancienne est brusquement transporté au vie siècle après J.-C., alors qu'il visitait la Rome mussolinienne. Il introduit dans cette Italie de l'Antiquité tardive, entre autres, l'utilisation du zéro, l'imprimerie, le journalisme à sensation, la poudre à canon, le téléscope. Craignant l'arrivée des «ténèbres" mentionnées par le titre, c'est-à-dire du Moyen Âge, il intervient dans la politique : il sauve le roi ostrogoth Théodat de son assassinat par Vitigès et, grâce à ses canons, empêche la reconquête de l'Italie par Bélisaire. Devenu conseiller privilégié des rois ostrogoths, il négocie d'égal à égal avec Justinien. À la fin du roman, il songe avec satisfaction que ses innovations lui survivront, quoi qu'il arrive: "L'histoire, sans conteste, avait été transformée. Les ténèbres étaient conjurées. »

3 Les auteurs de la postface font, à juste titre, le rapprochement avec Un Américain à la cour du roi Arthur de Mark Twain : on y trouve le même ton humoristique de journaliste, le goût pour la scène de comédie satirique (en particulier quand il s'agit de dépeindre des femmes capricieuses et des marchandages commerciaux ou politiques) auquel le lecteur d'aujourd'hui trouvera un charme désuet. En commun avec Mark Twain, encore, le positivisme qui s'amuse des superstitions (on notera les discussions de taverne entre orthodoxes, ariens, nestoriens et monophysites) et la conviction qu'avec 
du sens pratique et un esprit entreprenant, on vient à bout de tout. Les mêmes commentateurs ont donc beau jeu de s'amuser du caractère bien américain de ce personnage. Mais je crois que Sprague de Camp était moins naif qu'ils ne le pensent - il faut se garder de prendre un humoriste au pied de la lettre ${ }^{2}$ - et ce n'est certainement pas en toute innocence qu'il a intitulé Lest Darkness Fall une œuvre qui commence un soir d'orage, près du Panthéon, en 1938 ou 1939 : raconter les efforts de Martin Padway dans un $\mathrm{VI}^{\mathrm{e}}$ siècle plein des vestiges d'une brillante civilisation et qu'il voit tomber peu à peu dans les « ténèbres ", n'est-ce pas une manière de compenser l'impuissance dans laquelle se trouvait un Américain lucide visitant la vieille Europe au bord du conflit ${ }^{3}$ ? D'autre part, l'auteur manifeste une connaissance honorable de l'histoire, pourtant peu vulgarisée, de l'Italie ostrogothique. Si la peinture des personnages n'est pas toujours exempte de clichés, elle est la plupart du temps plutôt nuancée. Les Romains décadents ne sont pas si décadents qu'on s'y attendrait, les Ostrogoths sont rarement des barbares et Padway leur reconnaît les qualités de la bonne volonté et de la tolérance : il n'est pas sûr que les livres d'histoire contemporains de ce roman aient toujours été aussi équitables dans leurs jugements.

4 Le titre français du roman de Robert Silverberg, Les temps parallèles ${ }^{4}$, est tout à fait inapproprié : l'idée qu'il existe des univers parallèles permet à de nombreux auteurs de voyages dans le temps de se débarrasser de la difficulté que présentent les paradoxes temporels ${ }^{5}$. Or il n'est nullement question d'univers parallèles dans ce roman qui consiste au contraire en un jeu sur ces paradoxes. L'auteur imagine qu'au début du XXI siècle, l'on a découvert le moyen de voyager dans le passé. Cette invention a été aussitôt exploitée à des fins touristiques : on a institué un corps de " guides temporels " chargés d'escorter tous ceux qui veulent aller visiter les civilisations disparues et assister aux grands événements de l'Histoire - moyennant finances, bien entendu. Le roman raconte l'histoire de l'un de ces guides, Daniel Judson. Doctorant en histoire byzantine, il est recruté par le "Service temporel ». Après une formation théorique et un stage pratique dans la Louisiane des $\mathrm{XIX}^{\mathrm{e}}$ et $\mathrm{xx}^{\mathrm{e}}$ siècles, il s'installe à Istanbul pour se spécialiser dans le monde byzantin. Sous la conduite d'un guide confirmé nommé Métaxas, il apprend comment faire visiter la sédition Nika, par bonds successifs de quelques heures, de manière à tomber exactement aux moments forts de cet épisode. Le même Métaxas lui permet, à titre d'extra, de faire partie des innombrables jeunes gens recrutés pour passer une nuit avec Théodora: expérience décevante, car Théodora, bien qu'experte, fait l'amour de manière mécanique et sans âme. À son tour, il mène des groupes de touristes de 400 à 537 de manière à leur faire connaître en quelques jours l'histoire de la première Sainte-Sophie, de sa reconstruction sous Théodose II et de l'édification du sanctuaire voulu par Justinien. Il leur fait entendre les vraies paroles de l'empereur en cette occasion, fort éloignées du fameux " Ô Salomon, je t'ai surpassé ». Il leur fait encore assister à l'arrivée des croisés ou à la prise de Constantinople par les Turcs et à l'entrée de Mahomet II dans Sainte-Sophie. Il doit remédier à quelques imprudences commises par les personnes qu'il accompagne : une institutrice de l'Ohio veut offrir sa généreuse poitrine à Bohémond de Hauteville au moment où il entre dans la ville conquise, un agent de change pédophile s'échappe dans le temps pour assouvir ses instincts. En dehors de ses heures de travail, Daniel Judson se délasse chez son ami Métaxas qui s'est fait construire une agréable villa sous le règne d'Alexis Comnène; il y converse avec un professeur allemand qui écume les bibliothèques byzantines (celle d'Alexandrie est trop surveillée par l'implacable Patrouille du Temps) et qui en a rapporté « la Nausicaa et le Triptolème de Sophocle, ainsi 
que l'Andromède d'Euripide, les Pleiades, le Phaéton et l'ÆEdipe », réalisant ainsi le rêve de tous les hellénistes. C'est Métaxas, aussi, qui, apprenant que Daniel Judson est d'origine grecque par sa mère, l'incite à aller à la recherche de ses ancêtres. Il découvre ainsi qu'il descend d'une branche de la famille Doukas et tombe éperdument amoureux de Pulchérie, sa «multi-arrière-arrière-grand-mère », amour très sensuel et partagé. Malheureusement, cet amour interfère avec son activité professionnelle et il tombe dans un imbroglio de paradoxes temporels qui le voit se multiplier en plusieurs versions de lui-même.

5 Il existe une parenté entre ce roman et celui de Sprague de Camp : même point de vue "américain ", même orientation satirique. Mais la satire de Silverberg est beaucoup plus virulente à l'égard de ses compatriotes et très pessimiste. Les touristes sont ignares, avides de sensationnel et finalement surtout préoccupés d'eux-mêmes. Le milieu des guides temporels ne vaut guère mieux, ne s'intéressant qu'aux trafics illicites et aux aventures sexuelles ${ }^{6}$. Le héros, passé ses premiers émois de passionné d'histoire byzantine («Moi, Judson Daniel Elliott III, j'étais la tête nue sous le ciel de Byzance, ici, en l'an 408, tandis que l'empereur de Byzance passait devant moi en grande tenue ! [...] J'étais sidéré; et rien que par Arcadius. Que se serait-il passé s'il s'était agi de Justinien? de Constantin? d'Alexis?»), est bientôt contaminé par ce milieu, dans lequel il se fond, ayant oublié ses premiers centres d'intérêt. Il faut dire que ce roman, en partie à cause de cette noirceur, n'est pas le plus attirant de l'œuvre prolifique de cet auteur ${ }^{7}$. Bien que, visiblement, Silverberg porte un intérêt personnel à l'histoire byzantine et qu'il se soit documenté, c'est une Byzance touristique qui est ici présentée, une série de cartes postales où l'on voit s'agiter des personnages mesquins devant un décor de monuments et d'événements fameux, bref l'équivalent d'un roman d'aventures se déroulant dans des pays exotiques, à la seule différence que l'exotisme spatial y est remplacé par l'exotisme temporel.

$6 \quad$ Il en va bien autrement des deux volumes de La mosaïque de Sarance du romancier canadien anglophone Guy Gavriel Kay, intitulés respectivement Le chemin de Sarance et Le Seigneur des empereurs ${ }^{8}$. Il ne s'agit pas d'un voyage dans le temps et, au premier abord, il n'y est pas question de Byzance. Le roman est classé par l'éditeur comme relevant de la fantasy, mais, sauf ce qui concerne un épisode très délimité, il n'en présente aucune des caractéristiques (magie, êtres fabuleux, chevalerie). De fait, il ressemble plutôt à un roman historique, mais un roman historique utopique (au sens propre) et uchronique, puisqu'il se déroule en une époque et en des lieux imaginaires. Ce n'est même pas sur terre, comme nous l'indique - mais c'est la seule chose qui nous l'indique - le fait qu'il y ait deux lunes dans le ciel ${ }^{9}$. Néanmoins, c'est bien de l'Italie et de l'Empire byzantin au vi siècle qu'il s'agit ; Sarance, c'est Byzance, une Byzance rêvée et revisitée. L'intrigue est complexe, comme on peut l'attendre d'un ouvrage qui compte environ mille trois cents pages (dans l'édition «J'ai lu»), avec de multiples personnages et de multiples points de vue narratifs, si bien qu'il n'est pas facile de la résumer. Je ne donnerai donc qu'un bref aperçu.

7 Dans le premier volume, on suit le mosaïste Caius Crispus, dit Crispin, qui, à l'invitation de l'empereur Valerius II, quitte sa Batiare natale alors gouvernée par les Antae ${ }^{10}$, un peuple barbare qui l'a conquise, et se rend à la prestigieuse Sarance. Le voyage est long, par des contrées sauvages où il fait la rencontre de forces surnaturelles primitives ${ }^{11}$, mais il finit par atteindre sa destination, s'étant adjoint au passage ce que la narratologie appelle des «adjuvants »: un militaire de bonne volonté, un barbare et 
une jeune femme qu'il a sauvée de la prostitution. À Sarance, il travaille à décorer la coupole du nouveau « sanctuaire de la Sagesse de Jad » que l'empereur fait construire. Il découvre le monde des factions du Cirque et les courses de chars qui ont la faveur de tout le peuple. Impliqué malgré lui dans les complots de la Cour, il est séduit par la personnalité et l'exceptionnelle intelligence de l'empereur et de l'impératrice - surtout de cette dernière, Alixana, qui ne ressemble nullement à la Théodora de Silverberg (et de Procope). Le second volume délaisse le protagoniste du premier pour s'intéresser au destin de divers autres personnages: un médecin de l'empire oriental de Bassanie, puissance toujours en conflit avec Sarance, un cocher vedette, Alixana et l'empereur lui-même, menacé par un grave complot. On ne retrouve vraiment Crispin qu'à la fin du roman, quand il rentre dans son pays. L'œuvre s'achève sur une ingénieuse surprise dont il suffira de dire - pour éviter de la déflorer - qu'elle vient s'insérer dans l'histoire de l'art, non point de Sarance, mais de notre propre monde.

On aura compris que ce roman est d'une tout autre ampleur que les deux autres, et d'une grande richesse. Les personnages ne sont pas de simples silhouettes et l'auteur manifeste des ambitions littéraires et morales (pour ne pas dire philosophiques) indéniables. Cependant, pour un article figurant dans Anabases, plutôt que ces qualités, j'évoquerai le traitement de la matière byzantine par Guy Gavriel Kay. Le lien avec la Byzance réelle $\mathrm{du} \mathrm{VI}^{\mathrm{e}}$ siècle n'est nullement dissimulé. Dans ses "remerciements" placés en tête du premier volume, l'auteur définit son œuvre comme «ce qui veut être essentiellement un roman sur fond de culture byzantine». Il cite quelques-uns des travaux qu'il a utilisés, en particulier ceux d'Alan Cameron sur le Cirque et divers ouvrages sur l'esthétique et l'histoire de Byzance, et mentionne de nombreuses discussions avec des universitaires sur les "forums" électroniques consacrés à l'Antiquité tardive et à Byzance ${ }^{12}$. Bon nombre de lieux et de personnages réels sont aisément reconnaissables, sous le nom nouveau - souvent transparent - qui leur a été attribué. Il n'est donc pas nécessaire d'en donner la liste complète, mais on citera quelques exemples : Rhodias est Rome, Varéna Ravenne, la Trakésie la Thrace ; la reine Gisèle des Antae est Amalasonte (mais avec un destin plus heureux), le vieil empereur Apius Anastase, Valerius Ir Justin, Valerius II Justinien, Léontès Bélisaire et sa femme Styliane la fameuse Antonina de l'Histoire secrète, Pertennius d'Eubulus Procope de Césarée lui-même, peint sous un jour peu flatteur. G.G.K. l'accuse visiblement d'avoir assassiné la mémoire de Justinien ${ }^{13}$. On reconnaît sans hésitation la sédition " Nika ", les rivalités (on retrouve ici le sujet de Sprague de Camp) entre les chefs goths d'Italie après la mort de Théodoric, ici appelé Hildric, et le projet de reconquête de ce pays par Justinien. À mettre encore au compte de la fidélité au modèle, les deux récits de courses au Cirque sont particulièrement évocateurs : même le lecteur le moins intéressé par le «turf » et le sport sous toutes ses formes sera captivé par la manière dont l'auteur les fait revivre ; il comprendra enfin ce qu'était une course à Constantinople et pourquoi le peuple y mettait tant de passion. De même, la peinture de la vie des factions et de tous ceux qui gravitent autour des cochers, cuisiniers, hommes de peine, gestionnaires, danseuses et poètes, est très réussie, sans jamais sentir la fiche préparatoire, comme cela arrive souvent dans les romans historiques. Si G.G.K. n'échappe pas intégralement à l'anachronisme (mais peut-on parler d'anachronisme à propos d'un roman situé en nul temps ?) dans les situations et les dialogues ${ }^{14}$, le défaut reste très limité et plutôt de nuance que de fond, et ce n'est après tout que la loi du genre romanesque, car un roman qui respecterait strictement l'authenticité des points de vue ne ressemblerait guère à ce qu'on appelle aujourd'hui un roman et l'on doute qu'il parviendrait à trouver 
un public à notre époque. En réalité, l'auteur a mis tout son soin à prêter presque partout à ses personnages des connaissances et des conceptions de l'époque, en particulier dans le domaine des croyances, dussent-elles aller à l'encontre des idées reçues dans la nôtre ${ }^{15}$.

9 Mais le parti pris de situer l'intrigue dans un monde parallèle lui permet, outre d'éviter le reproche de l'anachronisme, de ne pas être prisonnier du carcan historique ${ }^{16}$. C'est ainsi qu'il peut condenser les siècles : pendant que Valérius règne à Sarance (donc au $\mathrm{VI}^{\mathrm{e}}$ siècle), dans le désert de Bassanie, un marchand d'âge mûr quitte une nuit la caravane dont il fait partie pour aller seul dans les sables à la rencontre d'une illumination qui changera le monde: on aura reconnu en cet Ashar ibn Ashar le prophète de l'sslam. De même, le successeur de Valérius impose déjà la rigueur iconoclaste à Sarance et fait casser les mosaïques du «sanctuaire de la Sainte Sagesse de Jad ». Un degré plus loin se situent les transpositions, dont la plus voyante est celle de la religion. L'empire sarentin, en effet, reconnaît un seul dieu, Jad, que l'on vénère dans des temples gérés par un clergé, avec un patriarche à Sarance et un autre isolé dans la vieille capitale occidentale de Rhodias. Mais il existe aussi une hérésie, qui a la faveur des Antae de Batiare et d'un certain nombre de Sarentins, dont l'impératrice Alixana elle-même : aux côtés de Jad figure son fils Helladikos, dont on commémore la chute dans la mer, car il est tombé pour sauver l'humanité. Les helladikiens le vénèrent à l'égal de son père et ont pour signe de reconnaissance le dauphin - c'est presque le poisson. On voit que, malicieusement, G.K.K. a inversé les rôles de vainqueur et de vaincu dans le conflit arien. Ailleurs, l'auteur modifie les personnages ou invente ni plus ni moins. Son Valérius est un mélange du véritable Justinien et d'un Justinien idéalisé bien différent du personnage historique : $\mathrm{s}$ 'il en conserve l'apparence physique, l'intelligence et l'énergie, il est un empereur modèle qui se soucie avant tout du bien de son empire, curieusement tolérant (il ne poursuit les helladikiens, est-il précisé, qu’à contrecœur, pour des raisons politiques) et d'une grande humanité. Son destin aussi est tout autre. De son côté, Gisèle ne périt pas, au contraire d'Amalasonte, mais fuit à Sarance, où elle finit par épouser Léontès/Bélisaire et devenir impératrice. Styliane a gardé de l'Antonina dépeinte par Procope la perversité, l'ambition politique et la sexualité débordante, mais elle n'est plus d'humble origine: sans doute G.G.K. a-t-il considéré qu'il n'avait pas besoin d'un doublet de Théodora (ce qu'elle est dans l'Histoire secrète) et qu'il valait mieux en faire, par contraste avec la danseuse parvenue au trône, l'héritière d'une grande famille, ennemie acharnée du couple impérial.

10 Paradoxalement, La mosaïque de Sarance, qui est infidèle à l'histoire, qui inverse et reconstruit délibérément la matière historique, fournit une évocation bien plus exacte du monde de l'Antiquité tardive et de l'Orient médiéval que les romans de Sprague de Camp et de Silverberg qui tâchent de respecter les données de l'histoire. Cette fausse Byzance est plus vraie que la «vraie » Byzance des voyageurs dans le temps, bref un exemple de ce «mentir vrai » que prônait Aragon. En guise de conclusion, il vaut sans doute la peine de citer un passage qui donnera une idée de la manière de G.G.K., surtout si on le compare au récit fourni par Procope ${ }^{17}$. Il s'agit du mot fameux de Théodora/ Alixana, par lequel elle rend courage à l'empereur au moment où celui-ci songe à fuir Byzance/Sarance devant l'émeute de la Victoire/sédition Nika. Un sénateur, Plautus Bonosus, revoit en pensée la scène à laquelle il a assisté :

11 «Tous les hommes présents étaient restés cois. Mais la seule femme présente avait pris la parole. 
préférerais mourir drapée de pourpre en ce palais, avait dit l'impératrice Alixana d'une voix égale, plutôt que de mourir de vieillesse en exil n'importe où ailleurs." Tandis que les hommes discutaient, elle s'était tenue à la fenêtre donnant sur le levant, contemplant la cité en flammes au-delà des jardins et des palais. Après s'être retournée, elle avait échangé un regard avec Valérius: "Tous les enfants de Jad naissent pour mourir. Les parures de l'Empire ne font-elles pas un suaire élégant, Monseigneur ?"

Bonosus se rappelait fort bien : le visage de Faustinus était devenu livide ; Gésius avait ouvert, puis refermé la bouche, l'air soudain vieilli, sa chair pâle et parcheminée marquée de rides profondes ${ }^{18}$. Et un autre souvenir, qui ne quitterait jamais Bonosus : l'Empereur auprès de son trône, et le brusque sourire qu'il avait adressé à la femme ravissante et menue à la fenêtre.

Avec un étrange chagrin, Plautus Bonosus s'était rendu compte, entre bien d'autres choses, qu'il n'avait jamais de sa vie regardé un homme ou une femme, ou reçu de quiconque un regard évoquant même de façon lointaine celui qu'adressait en retour à Valérius la danseuse devenue leur impératrice à tous ${ }^{19}$. "

\section{NOTES}

1. Titre original : Lest Darkness Fall, traduction française de Christian Meistermann publiée en 1972 par Marabout et reprise aux Belles Lettres en 1999 (coll. « Le cabinet noir », n²8), avec une postface d'Hélène et Pierre-Jean Oswald comprenant une brève notice sur l'auteur et son roman. On y apprend que cette œuvre parut d'abord en 1939 dans la revue de science-fiction Unknown (en feuilleton?), puis en librairie en 1949.

2. On peut citer cette phrase de Sprague de Camp qui résume le destin de Martin Padway : «Il ne connaîtrait jamais plus les joies ineffables de l'American Journal of Archaeology, de Mickey Mouse, des chasses d'eau, de la belle langue anglaise... »

3. Un certain nombre de détails sur la Rome contemporaine donnent à penser que Sprague de Camp a réellement visité la ville, et peut-être l'idée de son roman lui est-elle venue face au Panthéon, si bien conservé, comme s'il avait voyagé dans le temps depuis l'Antiquité jusqu'à nos jours.

4. Paru aux États-Unis en 1969 sous le titre Up the Line, traduction française de Henri-Luc Planchat, éditions du Bélial, 2004, repris en 2006 dans le Livre de Poche Science-fiction ( $\left.{ }^{\circ} 7282\right)$.

5. Le principal de ces paradoxes peut être présenté ainsi, comme le faisait Barjavel dans son Voyageur imprudent : un homme remonte dans le temps et assassine son père avant que celui-ci ait pu avoir un enfant. Donc, notre voyageur n'a jamais existé. Mais, s'il n'a jamais existé, il n'a pas pu remonter dans le temps et tuer son père. Donc, il existe, et il a pu tuer son père etc. Dans ce cas, la théorie des univers parallèles postule qu'après le meurtre du père, un nouvel univers a été créé, où le voyageur, en effet, n'a jamais existé. Mais son univers d'origine subsiste parallèlement. On peut aussi considérer qu'il n'est pas allé véritablement dans le passé, mais dans le passé d'un autre univers, où le père a été tué et, par ex., les Allemands ont gagné la Seconde Guerre mondiale (sujet d'un remarquable roman de Philip Dick), sans que cela remette en cause l'univers d'origine. 
6. On apprend au passage que les femmes de Byzance n'étaient pas si prudes qu'on les dépeint parfois. Tout le roman est d'un point de vue strictement masculin, la plupart des personnages féminins sont réduits à la plus simple expression, c'est-à-dire à leur attrait sexuel.

7. Ainsi s'explique sans doute le délai de trente-cinq ans qui sépare la publication aux Etats-Unis de la traduction française. On crédite R. Silverberg de plus de cent romans et nouvelles de Science-Fiction.

8. The Sarantine Mosaic: 1. Sailing to Sarantium, 1998; 2. Lord of Emperors, 2000. Traduction française d'Élisabeth Vonarburg parue en 2001 chez Buchet/Chastel, Pierre Zech, reprise en 2005 dans la coll. « J'ai lu».

9. L'auteur fournit en outre une carte qui dessine des côtes de nulle part, mais on peut y reconnaitre la silhouette - très déformée - de la Méditerranée orientale, de l'Italie, raccourcie et élargie, jusqu'aux rivages de la Palestine, l'Asie mineure ayant perdu son saillant et la Crète ayant fait un quart de tour sur elle-même.

10. Notre univers aussi a connu des Antae ou Antes, cités par Jordanès et Procope (dans ses Guerres gothiques) : c'est le premier peuple slave connu. Mais ils n'ont pas poussé jusqu'à la Méditerranée.

11. Ici se place l'épisode qui relève proprement de la fantasy auquel j'ai fait allusion précédemment.

12. L'auteur précise encore qu'il a emprunté son titre à un poème de Yeats, Sailing to Byzantium et l'épigraphe est tirée des Chroniques du voyage de Vladimir, Grand Prince de Kiev, à Constantinople.

13. Et ce point de vue est mené jusqu'à sa conséquence dernière, comme on le verra si on lit le roman.

14. Ainsi telle scène d'altercation dans une taverne, ou des conduites féminines qui sont plus proches de ce qui prévaut à notre époque que des convenances dans l'Antiquité tardive.

15. C'est le cas de la faible valeur accordée à la vie humaine ou de la croyance au surnaturel même chez les personnages les plus rationnels.

16. Cette autre planète et son autre Histoire, il y a situé précédemment un roman, intitulé en français Les lions d'Al Rassan (qui devrait, paraît-il, être prochainement adapté par Hollywood). Cette œuvre, moins réussie, à mon avis, que la Mosaïque de Sarance, se réfère à l'Espagne de la Reconquista, autour du personnage de Rodrigue de Bivar.

17. Bell. pers., I, 24, 37-41.

18. Faustinus et Gésisus sont respectivement le Maître des offices et le chancelier. Le second semble inspiré par le personnage de l'eunuque Narsès.

19. Le chemin de Sarance, trad. d'Élisabeth Vonarburg, p. 314-315 dans l'édition J'ai lu.

\section{AUTEUR}

\section{PIERRE-LOUIS MALOSSE}

Université Paul Valéry-Montpellier III

pl@malosse.org 